\title{
A PACIENTE GESTANTE: NA UNIDADE DE TERAPIA INTENSIVA
}

\author{
THE PREGNANT PATIENT IN INTENSIVE CARE UNIT
}

Antonio A. Nogueira' ${ }^{1}$ Francisco J.C. Reis ${ }^{1} \&$ Patrícia A.S. Reis ${ }^{2}$

\begin{abstract}
'Docentes, ${ }^{2}$ Médica Assistente. Departamento de Ginecologia e Obstetrícia da Faculdade de Medicina de Ribeirão Preto - USP Correspondência: Prof. Dr. Francisco J. C. Reis. Departamento de Ginecologia e Obstetrícia. Av Bandeirantes, 3900 - 8" andar, CEP 14049-900 - Ribeirão Preto - SP.
\end{abstract}

NOGUEIRA AA; REIS FJC \& REIS PAS. A paciente gestante na unidade de terapia intensiva. Medicina, Ribeirão Preto, 34: 123-132, abr./jun. 2001.

RESUMO: A internação de pacientes gestantes, em unidades de terapia intensiva (UTI), é rara, contudo, dentre tais pacientes, apresenta-se elevada mortalidade. O objetivo deste artigo é discutir alguns aspectos da fisiologia normal da gestante, assim como os principais aspectos do bem-estar fetal, de forma a tornar possível uma melhor abordagem das pacientes: gestantes gravemente enfermas. São discutidas ainda algumas situações como a pré-eclampsia, a embolia por líquido amniótico, o edema pulmonar induzido por tocolíticos, as hemorragias de causa obstétrica, as infecções de causa obstétrica e o trauma na gestação. O conhecimento das particularidades da gestação e do bem-estar fetal, assim como o de algumas situações específicas da gestação, é fundamental no sentido de se reduzir a mortalidade das pacientes gestantes internadas em UTI.

UNITERMOS: Gravidez. Unidades de Terapia Intensiva. Mortalidade Materna.

\section{INTRODUÇÃO}

Hoje, não há dúvidas que a mortalidade materna global está em declínio, contudo é preocupante que a mortalidade de gestantes internadas em UTI permaneça excessivamente alta $(10 \text { a } 20 \%)^{(1)}$. Em alguns centros especializados, essas pacientes são conduzidas por obstetras com experiência e treinamento em terapia intensiva, junto com outros especialistas, incluindo anestesiologistas e intensivistas com treinamento em obstetrícia, o que, na maioria dos serviços, não é possível e a condução dos casos fica a cargo de intensivistas gerais. A repercussão da pequena experiência, na condução das gestantes em estado crítico, é notada em várias publicações, por exemplo, Steinberg \& Farine $^{(2)}$, em uma revisão de 10 anos, no Canadá concluíram que $55 \%$ das mortes maternas ocorreram pela inadequação de cuidados, sendo elas, portanto, evitáveis. Em torno de 1/3 das mortes maternas, na Suécia, estão associadas a cuidados inferiores aos tidos como padrão ${ }^{(3)}$.

O propósito deste artigo é revisar alguns tópicos relacionados a enfermidades graves em gestantes, que frequentemente evoluem com necessidade de tratamento intensivo. Pretende-se oferecer ao intensivista a possibilidade de uma melhor compreensão de fenômenos ligados à gestação, assim como ao obstetra a de revisar alguns princípios de Medicina Intensiva aplicáveis à paciente grávida.

\section{MODIFICAÇÕES FISIOLÓGICAS E OR- GÂNICAS DURANTE A GRAVIDEZ}

Durante a gravidez, ocorrem diversas modificações adaptativas no organismo materno, e, dentre elas, parece-nos fundamental para a abordagem em terapia 
intensiva a discussão das modificações na fisiologia cardiovascular, respiratória, renal e gastrintestinal.

As mudanças adaptativas cardiocirculatórias têm o objetivo de maximizar o fluxo úteroplacentário e suprir as perdas decorrentes do parto. Ocorre aumento progressivo no volume sanguíneo, atingindo cerca de $40 \%$ no terceiro trimestre, o volume de plasma aumenta mais do que massa de células vermelhas, o que causa uma anemia relativa, com redução de cerca de $12 \%$ no hematócrito. A perda de 0,6 litros, que se segue no parto normal, e de 1,0 litros, após uma cesariana, é bem tolerada, sem mudança significativa no hematócrito. $\mathrm{O}$ aumento do volume sanguíneo acompanha-se de aumento do débito cardíaco de $30-50 \%$, que ocorre em função do aumento na freqüência cardíaca, do aumento no volume sistólico e da diminuição da resistência periférica ${ }^{(4)}$.

A pressão arterial sistêmica diminui, durante a gestação, até o início do terceiro trimestre, quando sofre ligeira elevação, permanecendo, contudo, em níveis inferiores aos pré-gestacionais. As resistências vasculares sistêmica e pulmonar estão diminuídas 20-30\%. As pressões venosa central e capilar pulmonar permanecem inalteradas ${ }^{(5)}$.

$\mathrm{O}$ útero aumentado pode comprimir a aorta e principalmente a veia cava inferior, o que causa a diminuição do retorno venoso e, em conseqüência do débito cardíaco, algumas vezes, associado com reflexo vasovagal. Na gestação a termo, o débito cardíaco aumenta até $20 \%$, quando a paciente passa da posição supina para o decúbito lateral esquerdo. Maiores alterações hemodinâmicas podem ocorrer durante o trabalho de parto, o débito cardíaco aumenta por aproximadamente $10-15 \%$ e, a cada contração uterina, ocorre a passagem de $300-500 \mathrm{ml}$ de sangue para a circulação sistêmica ${ }^{(6)}$.

A função pulmonar na gestação é afetada por mudanças nas vias aéreas, na caixa torácica e na mecânica respiratória. Nas vias aéreas, ocorrem hiperemia, edema da mucosa, hipersecreçao e friabilidade. Tais efeitos são predominantes no terceiro trimestre e causam sintomas secundários como epistáxis, obstrução nasal e mudanças na voz. Em pacientes grávidas, deve-se ter cuidado extra na inserção de sondas naso-gástricas e intubações ${ }^{(7)}$.

O útero gravídico eleva do diafragma com diminuição progressiva da capacidade respiratória residual funcional (CRF), em torno de 10-25\% no termo. Há, também, um aumento significante na ventilação, iniciando no primeiro trimestre e alcançando $20-40 \%$ no termo. Essa mudança é devida a dois fatores: o aumento na produção de $\mathrm{CO}_{2}$, de cerca de $34-50 \%$ no termo, e o aumento na frequiência respiratória devido a progesterona sérica, que age estimulando os centros respiratórios ou aumentando a sensibilidade dos mesmos a $\mathrm{PCO}_{2}$. O aumento ventilatório superior ao aumento na produção de $\mathrm{CO}_{2}$ leva a uma alcalose que é compensada pelo aumento na excreção renal de bicarbonato. $\mathrm{O} \mathrm{CO}_{2}$ arterial está reduzido a $28-32 \mathrm{mmHg}$ e o bicarbonato a $18-21 \mathrm{mEq} / \mathrm{L}$, o pH arterial é mantido de 7,40 a 7,47. O consumo de oxigênio aumenta 20 a $33 \%$ no terceiro trimestre, não somente devido às necessidades fetais mas, também, à demanda metabólica materna. A redução da CRF e o aumento nas necessidades de oxigênio, resultam em redução da reserva de oxigênio, levando a gestante a uma maior susceptibilidade à hipoxia, o que pode ocorrer rapidamente em resposta à hipoventilação ou apnéia ${ }^{(8)}$.

O fluxo sanguíneo renal aumenta 60 a $80 \%$ até o segundo trimestre, com algum declínio no terceiro trimestre. A taxa de filtração glomerular aumenta 50\% em torno da $16^{\mathrm{a}}$ semana e permanece elevada durante toda a gestação. Em consequiência, os níveis de creatinina variam de 0,5 a $0,7 \mathrm{mg} / \mathrm{dl}$. Valores de creatinina, considerados normais em pacientes não gestantes, podem indicar comprometimento renal na grávida. A dilatação da pélvis renal e do ureter, devido a mudanças hormonais ou efeitos mecânicos, pode aumentar a incidência de infecções urinárias, especialmente depois de instrumentação da bexiga ${ }^{(9,10)}$.

Na grávida, ocorre uma redução progressiva da pressão no esfíncter inferior do esôfago, talvez devido a um aumento na progesterona sérica. A posição do estômago é deslocada, levando a uma diminuição adicional na efetividade do esfíncter gastro-esofágico ${ }^{(11)}$. O trabalho de parto ativo e a administração de analgésicos dificultam ainda mais o esvaziamento gástrico, tornando a paciente mais suscetível à aspiração ${ }^{(12)}$. A mulher grávida deve, portanto, sempre ser considerada de risco para aspiração do conteúdo gástrico.

\section{OXIGENAÇÃO FETAL}

O fornecimento de oxigênio para a placenta e o feto é dependente do conteúdo de oxigênio arterial materno e do fluxo sanguíneo uterino. Normalmente, os vasos uterinos estão dilatados ao máximo; desta forma, uma redução no débito cardíaco materno afeta diretamente o fluxo úteroplacentário. A hipotensão materna e a ação de catecolaminas endógenas ou 
exógenas podem resultar em vasoconstricção da artéria uterina. O fluxo úteroplacentário também é reduzido, durante as contrações uterinas, de forma proporcional a sua intensidade. A alcalose materna afeta a oxigenação fetal tanto por redução do fluxo uterino quanto pelo desvio para a esquerda da curva de dissociação da hemoglobina materna. A administração de oxigênio à mãe está associada com melhora nos índices de bem-estar fetal ${ }^{(13)}$.

Apesar de a $\mathrm{pO}_{2}$, na veia umbilical, raramente exceder $40 \mathrm{mmHg}$, o conteúdo total de oxigênio no feto é elevado. Em função da presença da hemoglobina fetal, uma $\mathrm{pO}_{2}$ de $30-35 \mathrm{mmHg}$, na veia umbilical, leva a uma saturação de 80 a $90 \%$ da hemoglobina. Outras adaptações fetais incluem a concentração relativamente alta de hemoglobina $(15 \mathrm{~g} / \mathrm{dl})$ e o elevado débito cardíaco ${ }^{(14)}$.

A redução de até $50 \%$ do fluxo sanguíneo úteroplacentário é bem tolerada por períodos curtos, assim como a redução de $50 \%$ da oxigenação não leva a acidose fetal. Uma redução adicional resulta em metabolismo anaeróbio e redirecionamento do fluxo sanguíneo para o cérebro, coração e supra renais. O dano cerebral fetal irreversível ou a morte ocorrem com uma redução de $75 \%$ do conteúdo de oxigênio. Em uma situação previamente normal, isso não ocorre antes de uma interrupção de 10 minutos do fornecimento de $\mathrm{O}_{2}{ }^{(15)}$.

A melhoria da oxigenação fetal deve ser considerada no manejo de qualquer gestante em estado crítico. Dependendo da idade gestacional, o parto pode ser a melhor opção. Caso não o seja, o fornecimento de oxigênio deve ser ampliado, o que pode ser conseguido otimizando a oxigenação materna, melhorando a capacidade de transporte de $\mathrm{O}_{2}$, por transfusões e aumentando o débito cardíaco. O aumento da concentração de oxigênio inspirado causa pequena ele- vação na $\mathrm{pO}_{2}$ fetal (2-10mmHg), o que, contudo, resulta em uma grande mudança na saturação fetal de oxigênio. $\mathrm{O}$ fornecimento de oxigênio melhora o perfil acidobásico fetal, alivia as alterações anormais da freqüência cardíaca fetal e reverte a redistribuição do fluxo de sangue que acontece no feto em hipoxia ${ }^{(16)}$.

\section{AVALIAÇÃO DO BEM-ESTAR FETAL}

É necessário considerar que mudanças no bemestar fetal podem afetar as decisões no manejo da paciente grávida em estado crítico e que os diversos procedimentos para diagnóstico de terapêutica, rotineiros em UTI, podem implicar em comprometimento fetal. Tais fatos justificam o emprego de técnicas para a monitorização da vitalidade. Na Tabela I, apresentamos os principais métodos de investigação da vitalidade fetal assim como suas principais vantagens e limitações.

É consenso, atualmente, que, na avaliação da grávida em estado crítico, a investigação da vitalidade fetal, pode ser realizada com sucesso, empregandose a cardiotocografia e o perfil biofísico fetal (PBF) de rotina e ampliado. A cardiotocografia tem valor limitado antes da $32^{\mathrm{a}}$ semana por depender da maturidade neurológica fetal ${ }^{(17)}$. O PBF tem alta especificidade, com taxa de mortalidade menor que $0,1 \%$, se o PBF está entre 8 e $10^{(18)}$.

\section{PROCEDIMENTOS DE UTI DURANTE A GESTAÇÃO}

O manejo ideal da paciente em estado crítico implica na necessidade frequente da utilização de procedimentos radiológicos, de terapêutica medicamentosa, de ventilação mecânica, de monitorização he-

Tabela I - Métodos de avaliação da vitalidade fetal aplicáveis a gestantes em UTI

\begin{tabular}{|c|c|c|}
\hline Método & Vantagens & Limitações \\
\hline - Cardiotocografia & $\begin{array}{l}\text { - Simplicidade } \\
\text { - Elevada sensibilidade }\end{array}$ & $\begin{array}{l}\text { - Limitada em fetos de menos de } 32 \text { semanas, } \\
\text { por depender de maturação neurológica } \\
\text { - Baixa especificidade }\end{array}$ \\
\hline $\begin{array}{l}\text { - Perfil Biofísico Fetal } \\
\text { - (PBF) e PBF ampliado }\end{array}$ & - Elevada especificidade & - Necessidade de equipamento ultra-sonográfico \\
\hline - Status acidobásico & - Método mais preciso & $\begin{array}{l}\text { - Requer procedimento invasivo } \\
\text { - Risco de transmissão vertical de doenças }\end{array}$ \\
\hline
\end{tabular}


modinâmica invasiva e de procedimentos para ressuscitação cardiorrespiratória. Também é necessário considerar alguns aspectos particulares como a hipotensão supina e os efeitos da hipertermia e déficit nutricional sobre a gestação.

\subsection{Procedimentos radiológicos}

Embora a exposição à radiação, durante a gestação, possua riscos bem definidos, como o de malformações fetais e o de carcinogênese, procedimentos essenciais para a mãe não devem ser omitidos. A exposição a menos que 5 rads está associada a baixos riscos de complicações, de forma geral o risco de câncer na infância aumenta cerca de $0, \mathrm{I} \%$ a cada rad de exposição intra-útero; na Tabela II, está apresentada a quantidade aproximada de radiação para os procedimentos mais comuns em UTI.

\begin{tabular}{lc}
$\begin{array}{l}\text { Tabela II - Quantidade de exposição fetal à } \\
\text { radiação, segundo o procedimento utilizado }\end{array}$ \\
\hline Procedimento & $\begin{array}{c}\text { rads de } \\
\text { exposição fetal }\end{array}$ \\
\hline RX de tórax & 0,001 a 0,008 \\
RX abdominal sem blindagem & 0,29 \\
Angiografia via femoral & 0,22 a 0,37 \\
Tomografia da pelve & 5
\end{tabular}

\subsection{Utilização de medicamentos}

Na condução de pacientes críticos, há necessidade de se empregarem inúmeros medicamentos com risco potencial para o feto, principalmente antes da $12^{\mathrm{a}}$ semana de idade gestacional. Entre eles, merecem destaque as catecolaminas, os anti-hipertensivos, os anticoagulantes, o sulfato de magnésio, drogas utilizadas para sedação, analgesia e bloqueio neuromuscular.

Quando se utilizam catecolaminas no controle hemodinâmico da paciente grávida, a principal preocupação é o efeito sobre a circulação úteroplancentária. De forma geral, com exceção à efedrina, que demonstra efeitos tanto de elevação da pressão arterial materna quanto de aumento do fluxo úteroplacentário, todas as outras drogas vasoativas em uso levam a algum prejuízo para a circulação maternofetal. Contudo, a instabilidade hemodinâmica tem se mostrado mais danosa ao feto do que qualquer droga vasoativa utilizada. Em tal contexto, devem ser sempre consideradas, inicialmente, manobras não farmacológicas como restauração da volemia e posicionamento da paciente em decúbito lateral esquerdo ${ }^{(19)}$. Mas, quando os agentes farmacológicos forem necessários, devem ser utilizados da forma convencional.

O uso de anti-hipertensivos vasodilatadores é necessário em pacientes críticas principalmente na presença de doença hipertensiva específica da gestação (DHEG). Um risco associado ao uso dessas drogas é a hipotensão severa e a redução do fluxo sanguíneo úteroplacentário. A hidralazina, a nifedipina, o labetolol, e o nitroprussiato de sódio são as drogas mais utilizadas atualmente. A hidralazina, droga mais comumente utilizada na gestação, produz controle efetivo da pressão com administração endovenosa em pequenos bolos e pode aumentar o fluxo uterino. A nifedipina sublingual ou nifedipina oral é segura e efetiva no controle da hipertensão severa, o fluxo de sangue uterino parece não ser afetado, contudo a hipotensão profunda é descrita particularmente quando se associa nifedipina sublingual ao uso do sulfato de magnésio. Uma valiosa droga alternativa é o labetolol endovenoso, que produz controle da pressão sanguínea sem interferir com o fluxo úteroplacentário e ainda pode prevenir a ocorrência de arritmias ventriculares que ocorrem em algumas pacientes com DHEG. O nitroprussiato de sódio é efetivo em crises hipertensivas agudas, mas apresenta o risco fetal de toxicidade pelo tiocianato; contudo, é bastante valioso para o uso em curto prazo da hipertensão severa no período de periparto. Devem ser evitados inibidores de enzima conversora devido ao risco de desenvolvimento de disfunção renal fetal ${ }^{(20)}$.

Quando é necessária a administração de anticoagulantes, por exemplo, no tratamento do tromboembolismo pulmonar e na doença cardíaca, a droga de escolha é heparina, que não é associada com comprometimento fetal e seus efeitos maternos podem ser prontamente revertidos. O uso de terapêutica trombolítica, na gestação, foi descrito em alguns casos isolados. Quando tomadas as precauções adequadas, tanto a estreptoqumase quanto o ativador do plasminogênio tecidual podem ser usados, mesmo na gestação inicial.

Sulfato de magnésio é usado para prevenir e tratar crises convulsivas associadas a DHEG. Embora existam controvérsias e outras drogas, como fenitoína e benzodiazepínicos, sejam usadas em outras partes do mundo, o sulfato de magnésio é a terapia padrão na América do Norte. Os principais efeitos adversos incluem o bloqueio neuromuscular com paralisia respiratória e os distúrbios na condução cardíaca; tais efeitos podem ser revertidos pela administração de cálcio via parenteral ${ }^{(21)}$. 
Pouco se sabe sobre os efeitos de drogas para sedação prolongada, analgesia e bloqueio neuromuscular sobre a gestação. Apenas a sedação com benzodiazepínicos foi associada com um pequeno risco de malformações congênitas, principalmente lábio leporino e fenda palatina, quando usada em gestação inicial $^{(22)}$.

\subsection{Ventilação mecânica}

Embora as mudanças fisiológicas respiratórias na gestação sejam bem compreendidas, poucos estudos existem a respeito dos efeitos da ventilação mecânica prolongada, para o manejo da falência respiratória, sobre a gravidez. As indicações para entubação e ventilação mecânica são semelhantes aquelas que ocorrem na paciente não gestante.

É consenso, no entanto, que o procedimento de entubação é crítico na gestante. As mudanças fisiológicas próprias da gestação implicam em alguns cuidados adicionais na técnica de intubação, a hiperemia e o edema das vias aéreas resultam em maior susceptibilidade ao trauma mecânico, devendo-se preferir a via orotraqueal ao invés da nasotraqueal ${ }^{(23)}$. A CRF diminuída e o aumento no consumo de oxigênio reduzem a reserva de oxigênio de forma que quedas na saturação arterial significante acontecem em períodos relativamente pequenos de apnéia durante a entubação, sendo a oxigenação prévia, portanto, essencial para evitar maiores danos.

\subsection{Monitorização hemodinâmica}

Tem ocorrido um aumento na utilização do cateter na artéria pulmonar para monitorização hemodinâmica em gestantes, na última década. As principais indicações incluem oligúria, DHEG severa, edema pulmonar, doença cardíaca severa, a síndrome da angús- tia respiratória do adulto, choque séptico e embolia por Iíquido aminiótico. A monitorização hemodinâmica invasiva é particularmente valiosa nessas pacientes, durante o trabalho de parto, quando ocorrem marcantes modificações hemodinâmicas.

A técnica de inserção do cateter na artéria pulmonar não é diferente da de outros pacientes críticos, mas a incidência de complicações pode ser menor já que as gestantes, geralmente, são jovens e sem enfermidades associadas. Conhecer as mudanças cardiovasculares fisiológicas é necessário para a correta interpretação dos dados obtidos. A Tabela III resume os achados hemodinâmicos normais na gestação ${ }^{(24,25,26)}$.

\subsection{Ressuscitação cardiopulmonar}

As mudanças fisiológicas cardiovasculares e respiratórias possuem um efeito significante na ressuscitação cardiopulmonar (RCP), durante a gestação. O aumento na taxa metabólica, a diminuição da reserva de oxigênio e a necessidade de débito cardíaco mais alto devem ser considerados. Os principais eventos que levam à parada cardíaca, na gestação, incluem a embolia por Iíquido aminiótico, embolia pulmonar, cardiomiopatias, complicações anestésicas, superdose de magnésio e infarto do miocárdio.

O manejo da parada cardíaca, na paciente grávida, segue os protocolos padrões com algumas modificações. A massagem cardíaca pode ser tecnicamente difícil na gestação avançada devido ao tamanho do útero e a ressuscitação na posição supina pode levar à compressão da veia cava inferior e da aorta, com conseqüente redução do débito cardíaco. É importante saber que uma força de compressão efetiva do tórax pode ser gerada com a paciente em uma posição de decúbito lateral esquerdo ${ }^{(27)}$.

Tabela III - Parâmetros hemodinâmicos durante a gestação e modificações em relação à paciente não gestante

\begin{tabular}{lll}
\hline & Valor médio & \multicolumn{1}{c}{$\begin{array}{c}\text { Modificação em relação } \\
\text { à não gestante }\end{array}$} \\
\hline Freqüência cardíaca & $83 \pm 10 \mathrm{BPS}$ & Aumento de $10 \%$ a $30 \%$ \\
Pressão arterial média & $90 \pm 6 \mathrm{mmHg}$ & Não muda \\
Pressão venosa central & $4 \pm 2,5 \mathrm{mmHg}$ & Não muda \\
Débito cardíaco & $6,2 \pm 1,0 \mathrm{l} / \mathrm{min}$ & $30 \%$ a $50 \%$ de aumento \\
Resistência vascular sistêmica & $1200 \pm 260$ dyne cm s & $20 \%$ a 30\% de diminuição \\
Resistência vascular pulmonar & $75 \pm 22$ dyne $\mathrm{cm} \mathrm{s}^{-5}$ & $20 \sim$ a 30\% de diminuição
\end{tabular}


A cardioversão elétrica e a defibrilação podem e ser realizadas durante a gestação, tomando-se o cuidado de remover o monitor fetal antes do procedimento para evitar a formação de um arco de corrente. $\mathrm{E}$ a terapia farmacológica deve ser utilizada conforme a indicação independente da gestação. A cesariana pós morte pode ser indicada quando tentativas iniciais de ressuscitação falharem em uma mulher com gestação de mais de 24-26 semanas, o tempo entre a interrupção da massagem cardíaca efetiva e o nascimento é o principal fator de prognóstico fetal ${ }^{(28)}$. A sobrevida sem seqüelas neurológicas do feto é melhor quando o procedimento é realizado dentro de 4 min da massagem cardíaca. Os esforços de ressuscitação devem ser continuados durante a cesariana para manter a máxima perfusão uteroplacentária. A realização de uma cesarea pós morte pode ainda, em algumas situações, inverter a compressão da aorta e cava e permitir ressuscitação bem sucedida da mãe e da criança.

\subsection{Hipotensão supina, hipertermia e nutrição}

Vários outros aspectos na condução da gestante em estado crítico diferem do manejo habitual na UTI; sendo os mais importantes a síndrome da hipotensão supina, os efeitos fetais da hipertermia e os aspectos nutricionais. Na posição supina, o útero grávido pode comprimir a veia cava, reduzindo o retorno venoso central, com resultante diminuição do débito cardíaco e pressão arterial. A compressão concomitante da aorta pode afetar o fluxo de sangue para o útero e causar hipoxia fetal, portanto é particularmente importante evitar esta posição em gestantes hemodinamicamente instáveis ou com sofrimento fetal; a paciente deve ser posicionada no lado esquerdo ou, pelo menos, com o quadril ligeiramente elevado.

Alguns estudos sugerem que hipertermia pode causar defeitos do tubo neural durante o primeiro trimestre e, principalmente, com temperaturas superiores a $39^{\circ} \mathrm{C}^{(29)} \mathrm{O}$ tratamento ativo de febre na gestante com esfriando externo ou acetaminofeno é apropriado.

A desnutrição pode resultar em retardo do crescimento intra-uterino (RCIU) e óbito fetal. O RCIU antes das 26 semanas de gestação pode levar a deterioração neurológica. As exigências nutricionais da mãe durante a gestação não são muito diferentes das do estado de não gestante, as exigências de calorias só aumentam por aproximadamente $300 \mathrm{kcal}$ por dia, e o controle da glicemia materna deve ser mantido de forma a evitar hiperglicemia e seus efeitos sobre o feto. O aporte de proteínas deveria ser aumentado por: 20$50 \%$ para apoiar o aumento da síntese de proteína materna e fetal. Só um número pequeno de nutrientes necessita ser aumentado na gestação; ferro, folato, e cálcio necessitam suplementação de 150-200 \% em relação à não gestante. Quando necessário, o uso de nutrição parenteral total tem se mostrado seguro durante a gestação com atenção especial para os cuidados mencionados $\operatorname{acima}^{(30)}$.

\section{CAUSAS OBSTÉTRICAS DE INTERNA- ÇÕES EM UTI}

Existem várias indicações para internação de pacientes grávidas em UTI, podendo estas ser divididas em causas obstetrícias e causas não obstétricas. As principais causas associadas à gestação são: a DHEG, a embolia por líquido amniótico, a hemorragia de causa obstétrica, a sepse de causa obstétrica.

\subsection{Doença hipertensiva específica da gestação}

A DHEG é uma desordem induzida pela gestação, caracterizada por alterações no endotélio vascular materno, relacionadas a um desequilíbrio entre substâncias vasodilatadoras e vasoconstrictoras. A vasocon-tricção arteriolar e volume plasmático reduzido, característicos da doença, podem levar a hipoperfusão de diversos órgãos e consequiente falência dos mesmos ${ }^{(31)}$.

Da perspectiva de cuidados intensivos, a DHEG pode levar rapidamente ao desenvolvimento de quadros mais graves. As crises convulsivas são as complicações neurológicas mais frequentes e definem a eclampsia. A síndrome HELLP, caracterizada por disfunção hepática, coagulopatia e hemólise (hemólise, enzimas hepáticas elevadas e plaquetas reduzidas), é uma variante associada com elevada morbidade ${ }^{(32)}$. Outras complicações maternas incluem edema pulmonar, disfunção renal, ruptura hepática, edema e hemorragia cerebral, e coagulação intravascular disseminada $^{(33,34)}$. A DHEG representa uma importante causa de morbimortalidade materna, sendo responsável por cerca de $20-50 \%$ das admissões obstétricas em UTI e por $12-17 \%$ de todas as mortes de maternas nos Estados Unidos ${ }^{(19)}$.

Do ponto de vista do obstetra, o manejo envolve o reconhecimento precoce da doença e o planejamento do parto. O tratamento é de suporte, não existem tratamentos efetivos que não a remoção do feto e da placenta ${ }^{(33)}$. Mais preocupante é a condução da paciente em uma fase precoce da gestação, quando a viabilidade do feto é questionável ou se apresenta instável. Na decisão de prolongar a gestação com o in- 
tuito de melhorar os resultados perinatais, devem sempre ser levados em conta os riscos maternos. O parto, geralmente, é aconselhável na presença de sinais de DHEG severa (Tabela IV).

A paciente com DHEG deve ser avaliada quanto à severidade da doença e à presença de complicações maternas ou fetais. Em termos de complicações maternas, é extremamente importante a avaliação cardiopulmonar, neurológica e laboratorial. A última inclui avaliação de função renal, estado de coagulação, e função hepática. Devido à natureza da doença, pacientes podem ser admitidos na UTI sem um quadro clínico e laboratorial típico. O intensivista deve estar atento para o diagnóstico diferencial com outras anomalias como trombocitopenia, deficiência orgânica hepática, e insuficiência renal.

Em UTI, há necessidade frequente do uso parenteral de anti-hipertensivos para controle de crises hipertensivas agudas e prevenção de danos vasculares maternos; nessa situação, a maior experiência é com hidralazina intravenosa, mas o labetalol intravenoso e a nifedipina sublingual ou oral também são seguros e efetivos. Baixas doses repetidas são preferíveis por evitar hipofluxo placentário e a reposição volemica cautelosa é, as vezes, necessária para controle de hipotensão secundária ${ }^{(35)}$. O nitroprussiato de sódio pode ser usado em crises agudas, mas devido à toxicidade fetal potencial deve ser minimizada a duração de sua infusão ${ }^{(36)}$. Depois do nascimento do feto, já não se aplicam tais restrições.

Algumas controvérsias existem a respeito da fluidoterapia na DHEG; ao mesmo tempo em que as pacientes apresentam depleção do volume intravascular $^{(37)}$. a reposição volêmica pode ser difícil devido à redução da pressão coloidosmótica plasmática e ao aumento da permeabilidade capilar, que poderia levar a edema cerebral secundário, à infusão de cristalói- $\operatorname{des}^{(38)}$. No período pós-parto imediato, existe ainda o risco de edema agudo pulmonar em função da redução na pressão coloidosmótica do plasma que acontece durante trabalho de parto e o aumento no volume de sangue central com as contrações uterinas ${ }^{(39)}$. A monitorização hemodinâmica invasiva está indicada na presença de hipertensão severa, refratária à terapêutica convencional, edema de pulmonar, oligúria e intervenções cirúrgicas ${ }^{(40)}$.

Existem várias complicações graves que podem ocorrer associadas a DHEG, sendo as mais importantes a eclampsia e o edema cerebral, a disfunção renal, o edema pulmonar, e a síndrome HELLP. As crises convulsivas ocorrem por uma conjunção de fatores vasoespasmo, isquemia, edema cerebral e encefalopatia de hipertensiva. $\mathrm{O}$ manejo envolve as medidas habituais para crises convulsivas, tratamento da DHEG, terapêutica anticonvulsivante, e resolução da gestação. A maioria dos casos responderá a tratamento com sulfato de magnésio, mas, se as crises persistem, podem ser necessários outros anticonvulsivantes ${ }^{(35)}$. $\mathrm{O}$ edema cerebral isolado é raro na DHEG, mas ,quando ocorre, é causa importante de mortalidade. Acreditase que tal edema aconteça secundário à hipoxia resultante da hipertensão severa, mas a excessiva reposição hídrica para tratar oligúria e a redução da pressão coloidosmótica são fatores que podem contribuir ${ }^{(38)}$. O manejo adequado inclui controle da pressão arterial, das crises convulsivas, correção da hipoxia e da hipercapnia e hiperventilação mecânica.

Na DHEG, pode ocorrer disfunção renal devido aos efeitos da depleção do volume, do vasoespasmo e da lesão endotelial (endoteliose glomerular). A gravidade da lesão se correlaciona até certo ponto com o grau de proteinúria. Como com outras manifestações de DHEG, a reversão do quadro é facilitada pela resolução da gestação ${ }^{(33)}$.

Tabela IV - Sinais e sintomas de pré-eclampsia severa

Pressão sangüínea

Proteinúria

Contagem: de plaquetas

Outros sistólica > $160 \mathrm{mmHg}$ e diastólica > $110 \mathrm{mmHg}$

$2 \mathrm{~g} / 24 \mathrm{~h}$ ou $100 \mathrm{mg} / \mathrm{dl}$ (amostra)

$<100.000 / \mathrm{ml}$

Dor epigástrica-ou em quadrante superior direito do abdome

Distúrbios visuais cefaléia e perda da consciência

Convulsões

Edema pulmonar

Elevação de enzimas hepáticas 
A síndrome HELLP é uma complicação da DHEG, caracterizada por deficiência de múltiplos órgãos(32) onde, provavelmente, a lesão endotelial seja o principal fator. O papel da CIVD, em sua patogênese, é controverso, e embora ela venha demonstrada em mais de 38.\% das pacientes, frequentemente é secundária ao descolamento placentário, à sepse, ou à morte fetal. A hipoperfusão hepática resulta em necrose periportal, elevação de enzimas, e ocasionalmente ruptura hepática. A resolução da gestação está indicada em qualquer idade gestacional ${ }^{(41)}$.

\subsection{Embolia por líquido amniótico}

Durante o parto, pequenas quantidades de líquido amniótico entram em contato com a circulação materna sem causar problemas, contudo, em raras ocasiões (1:8000 a 1:80000 nascimentos), pode ocorrer um quadro catastrófico conhecido como síndrome da embolia por Iíquido amniótico. O quadro clínico descrito como clássico envolve dispnéia e hipotensão seguida de convulsões e parada cardiorrespiratória. A mortalidade atinge até $86 \%$ dos casos. No momento da embolização, $40 \%$ dos fetos já foram a óbito e ocorreram cerca de $50 \%$ de descolamento de placenta. O óbito ocorre, geralmente, nas primeiras horas $(25 \%$ a $50 \%$ ), as pacientes que sobrevivem à primeira fase desenvolvem CIVD e a maioria apresenta graves lesões neurológicas ${ }^{(42)}$.

O tratamento deve considerar a manutenção da oxigenação e o suporte hemodinâmico, em função da gravidade do quadro a monitorização hemodinâmica invasiva, é fundamental e deve guiar a reposição volêmica e a administração de drogas vasoativas.

\subsection{Hemorragias de causa obstétrica}

As hemorragias de causa obstétrica, desencadeadas tanto por fatores prévios ao parto, como descolamento prematuro da placenta e a rotura uterina, quanto após o parto, como na atonia uterina ou ainda o desenvolvimento de CIVD, são uma causa importante de morbidade e mortalidade materna. O manejo ideal implica em medidas de suporte e medidas para conter a hemorragia. É necessário prever a coagulopatia dilucional pelo pronto fornecimento de sangue e pela instituição de medidas urgentes a contenção da hemorragia (massagem uterina e uso de ocitócitos, curetagens uterinas, histerectomia, até medidas heróicas como a ligadura das artérias hipogastricas).

\subsection{Edema pulmonar, induzido por tocolíticos}

Drogas betadrenérgicas, frequentemente utilizadas para inibição do trabalho de parto prematuro, podem levar ao edema agudo do pulmão, geralmente, por mecanismos multifatoriais, que incluem aumento da frequiência cardíaca, disfunção miocárdica e redução da pressão coloidosmótica secundária à hiperidratação. Alguns fatores como gestação múltipla, infecção materna e utilização prévia de corticosteróides podem aumentar o risco ${ }^{(43)}$.

$\mathrm{O}$ reconhecimento precoce e a instituição de cuidados terapêuticos com a interrupção da administração da droga betadrenérgica, o fornecimento de oxigênio e a utilização de diuréticos são passos fundamentais na condução bem sucedida de tais casos. Com tais medidas a mortalidade é hoje bastante rara.

\subsection{Infecções de causas obstétricas}

Durante o ciclo gravidopuerperal podem ocorrer situações como: amniorrexe prolongada, retenção placentária, instrumentação urinária e aborto provocado, que podem levar a infecções graves, inclusive com choque séptico. O manejo do choque séptico segue os princípios gerais, lembrando que a antibioticoterapia inicial deve cobrir gram-positivos, gram- negativos e anaeróbios.

As pacientes com deterioração do estado geral, apesar da antibioticoterapia, devem ser investigadas quanto à presença de microorganismos resistentes, tromboflebite pélvica, miometrite e abscessos localizados. Nas duas úItimas situações, pode ser necessária intervenção cirúrgica. É fundamental que não se retarde a indicação de intervenção cirúrgica e a monitorização de parâmetros, como a contagem de plaquetas, função renal e parâmetros respiratórios, podem ser úteis na definição do melhor momento para tal indicação.

\subsection{Trauma na gestação}

A conduta médica na grávida, vítima de trauma, deve considerar, além das peculiaridades fisiológicas da gestante, a existência de uma possível segunda vítima, o feto. A gestação aumenta a susceptibilidade para lesões abdominais, o aumento uterino o torna cada vez mais vulnerável à lesões. A partir da $12^{\mathrm{a}}$, semana de gestação, há ainda um aumento no risco de lesões da bexiga.

O bem-estar fetal está diretamente relacionado com a severidade do trauma e com o grau de comprometimento hemodinâmico materno, sendo as principais causas de mortalidade fetal, na grávida vítima 
de trauma, a morte materna e o descolamento prematuro da placenta, que pode ocorrer em até $20 \%$ dos casos de traumas maiores ${ }^{(44)}$.

O manejo inicial obedece aos preceitos do Advanced Trauma Life Suport (ATLS), considerando-se as alterações fisiológicas da gestante, fornecendo-se $\mathrm{O}_{2}$ suplementar, e, sempre que possível, deixando a paciente em decúbito lateral esquerdo.

\section{CONCLUSÕES}

A internação de mulheres durante o ciclo gravidopuerperal em unidades de tratamento intensi- va é rara, contudo, mesmo se tratando de pacientes jovens e geralmente sem patologias de base, a mortalidade é desproporcionalmente elevada em praticamente todo o mundo. Não menos preocupante é o fato de que a maioria dessas mortes poderia ser evitada pelo diagnóstico precoce da condição crítica e instituição da terapêutica adequada. Neste sentido, é fundamental que tanto o intensivista quanto o obstetra se preocupem em conhecer mais profundamente as afecções graves que podem ocorrer durante esse período da vida da mulher, assim como a melhor abordagem para as mesmas, levando-se em conta as modificações; fisiológicas próprias da gestação e o bem-estar fetal.

NOGUEIRA AA; REIS FJC \& REIS PAS. The pregnant patient in intensive care unit. Medicina, Ribeirão Preto, 34: 123-132, april/june 2001.

ABSTRACT: Intensive Care Unit (ICU) admission of pregnant patient is rare, however, the mortality rate of obstetric patients admitted to the ICU is high. The objective of this paper is to present some aspects of the physiologic changes in pregnancy and the most important points of the fetal well-being in order to provide the best management for the critically ill pregnant patient. Several topics were presented such as preeclapsia, amniotic fluid embolism, tocolitic-induced pulmonary edema, obstetric hemorrhage, obstetric infections and trauma in pregnancy. The knowledge of the pregnant patient and fetal physiology as well as the obstetric complications are very important in order to avoid maternal mortality due suboptimal standard of care.

UNITERMS: Pregnancy. Intensive Care Units. Maternal Mortality.

\section{REFERÊNCIAS BIBLIOGRÁFICAS}

1 - KILPATRICK SJ \& MATTHAY MA. Obstetric patients requiring critical care: a five-year review. Chest 101:1407-1412, 1992.

2 - STEINBERG WM \& FARINE D. Maternal mortality in Ontario from 1970 to 1980. Obstet Gynecol 66: 510-512, 1985.

3 - HOGBERG U; INNULA E \& SANDSTROM A. Maternal mortality in Sweden, 1980-1988. Obstet Gynecol 84: 240-244,1994.

4 - UELAND K. Maternal cardiovascular dynamics, VII: intrapartum blood volume changes. Am J Obstet Gynecol 126: 671-677,1976.

5-ROBSON SC; HUNTER S; BOYS RJ \& DUNLOP W. Hemodinamic changes during twin pregnancy: a doppler and M-mode echocardiographic çtudy. Am J Obstet Gynecol 161:12731278,1989 .

6 - MASHINI IS; ALBAZZAZ SJ; FEDEL HE; ABDULLA AM; HADI HA; HARP R \& DEVOE LD. Serial nonivasive evaluation of cardiovascular hemodynamics during pregnancy. Am J Obstet Gynecol 156:1208-1213,1987.
7 - CAMANN WR \& OSTHEIMER GW. Physiological adaptations during pregnancy. Int Anesthesiol Clin 28: 2-10, 1990.

8 - ARCHER GW \& MARX GF. Arterial oxygen terision during apnoea in parturient woman. $\mathbf{B r} \mathbf{J}$ Anaesth 46: 358360,1974 .

9 - MARCHANT DJ. Alterations in anatomy and function of the urinary tract during pregnancy. Clin Obstet Gynecol 21: 855-862,1978.

10 - MATTINGLY RF \& BORCOWF HI. Clinical implications of ureteric reflux. Clin Obstet Gynecol 21: 863-873, 1978.

11-VAN THIEL DH; GAVALER JS; JOSHI SN; SARA RK \& STREMPLE J. Heathburn of pregnancy. Gastroenterology 72: 666-668,1977.

12 - NIMMO WS; WILSON J \& PRESCOT LF. Narcotic analgesics and delayed gastris emptying during labour. Lancet 1: 890893,1975 .

13 - BARTNICKI J \& SALING E. The influence of rriaternal oxygen administration on the fetus. Int J Gynaecol Obstet 45: 8795,1994 . 
14 - MESCHIA G. Supply of oxygen to the fetus. J Reprod Med 23:160-165,1979.

15 - MYERS RE. Two patterns of perinatal brain damage and their conditions of occurence. Am J Obstet Gynecol 112: 246276,1972

16 - ARDUINI D; RIZZO G; MANCUSO S \& ROMANINI. Short-term effects of maternal oxygen administration on blood flow velocity waveforms in healthy and growth retarded fetuses. Am J Obstet Gyneco1159: 1077-1080,1988.

17 - NATALE R; NASELLO-PATERSON C \& TURLIUK R. Longitudinal measurements of fetal breathing, body movements, heart rate, and heart rate accelerations and desaccelerations at 24 to 32 weeks gestation. Am J Obstet Ginecol 151: 256263, 1985.

18 - MANNING FA; BASKETTITF; MORRISON I \& LANGE I. Fetal biophysical profile scoring; a prospective study in 1,184 high-risk patients. Am J Obstet Gynecol 140:289293,1981 .

19 - LAPINSK SE; KRUCZYNSKI K \& SLUTSKYI. Critical care in the pregnant patient. Am J Respir Crit Care Med 152: 427-445,1995.

20 - SHOTAN A; WIDERHORN J, HURST A \& ELKAYAM U. Risks of angiotensin-converting enzyme inhibition during pregnancy: experimental and clinical evidence, potential mechanisms and recomendation for use. Am J Med 96: 451-456, 1994.

21 - BEM-AMI M; GILADI Y \& SHALEV E. The combination of magnesium sulphate and nefedipine: a cause of neuromuscular blockade. Br J Obstet Gynaecol 101: 262-263, 1994.

22- BRIGGS GG; FREEMAN RK \& YAFFE SJ. Drugs in pregnancy and lactation. Williams and Wilkens, Baltimore 1986.

23 - CHEEK TG \& GUTSCHE BB. Maternal physiologic alterations during pregnancy. In: SHINIDER SM \& LIVINSON G, eds. Anesthesia for obstetrics. Williams \& Wilkins, Baltimore, p. 3-13,1987.

24-CLARK SL; COTTON DB; LEE W; BISHOP C; HILL T;SOUTHWICK J; PIVARNIK J; SPILLMAN T; DEVORE GR \&PHELAN J. Central hemodynamic assessment of normal term pregnancy. Am J Obstet Gynecol 161: 11439-1442, 1989.

25 - WALLENBURG HCS. Invasive hemodynamic monitoring in pregnancy. Eur J Obstet Gynecol Reprod Biol 42: S451$5454,1991$.

26-AMERICAN COLLEGE OF OBSTETRICIANS AND GYNECOLOGISTS. Invasive hemodynamic monitoring in obstetrics and gynecology: ACOG Technical Bulletin number 175 . Int J Gynecol Obstet 42: 199-205, 1993.

27- RESS GAD \& WILLIS BA. Resuscitation in late pregnancy. Anaesthesia 43: 347-349, 1988

28 - STRONG TH \& LOWE RA. Perimorten cesarean section. Am J Emerg Med 7: 489-494, 1989.

29 - HARVEY MA; MCRORIE MM \& SMITH DW. Suggested limits to the use of the hot tub and sauna by pregnant women. Can Med Assoc J 125: 50-53, 1981.
30 - LEVINE MG \& ESSER D. Total parenteral nutrition for the treatment of severe hyperemesis gravidarum: maternal nutritional effects and fetal outcome. Obstet Gynecol 72: 102-107, 1988.

31 - LINDHEIMER MD \& KATZ AI. Preeclampsia: pathophisiology, diagnosis, and management. Annu Rev Med 40: 233-250, 1989.

32 - WEINSTEIN L. Syndrome of hemolysis, elevated liver enzymes, and low platelet count: a severe consequence of hypertension pregnancy. Am J Obstet Gynecol 142: 159167, 1982.

33- NATIONAL HIGH BLOOD PRESSURE EDUCATION PROGRAM. Working Group. Report on high blood pressure during pregnancy. Am J Obstet Gynecol 163: 1689-1712, 1990.

34- REDMAN CWG \& ROBERTS JM. Management of pre-eclampsia. Lancet 341:1451-1454, 1994.

35 - DILDY GA \& COTTON DB. Management of severe preeclampsia and eclampsia. Crit Care Clin 7: 829-864, 1991.

36 - STRAUS RG; KEEFER JR; BURKE T \& CIVETTA JM. Hemodynamic monitoring of cardiogenic pulmonary edema complicating toxemia of pregnancy. Obstet Gynecol 55: 170$174,1980$.

37 - GALLERY EDM; HUNYOR SM \& GYORY AZ. Plasma volume contraction: a significant factor in both pregnancy-associated hypertension (pre-eclampsia) and chronic hypertension in pregnancy. Q J Med 48: 593-602, 1979.

38 - BENEDETTI TJ \& QUILLIGAN EJ. Cerebral edema in severe pregnancy-induced hypertension. Am J Obstet Gynecol 137: $860-862,1980$.

39-BENEDETTI TJ; KAIES R \& WILLIAMS W. Hemodynamic observations of severe preeclampsia complicated by pulmonary edema. Am J Obstet Gynecol 152: 330-334, 1985.

40 - CLARK SL \& COTTON DB. Clinical indications for pulmonary artery catheterization in the patient with severe preeclampsia. Am J Obstet Gynecol 158: 453-458, 1988.

41 - SIBAI BM. The HELLP syndrome (hemolysis, elevated liver enzymes, and low platelets): much ado about nothing. Am J Obstet Gynecol 162: 311-316, 1990.

42 - MORGAN M. Amniotic fluid embolism. Anaesthesia 34: 2022,1979

43 - PISANI RJ \& ROSENOW EC. Pulmonary edema associated with tocolitic therapy. Ann Intern Med 110: 714-718, 1989.

44- ROTHENBERGER D; QUATTLEBAUM FW; PERRY JF;ZABEL J \& FISCHER RP. Blunt maternal trauma: a review of 103 cases. J Trauma 18: 173-179, 1978.

Recebido para publicação em 06/07/2000

Aprovado para publicação em 04/05/2001 